\title{
Association Between Obesity and Short-And Long-Term Mortality in Patients With Acute Respiratory Distress Syndrome Based on the Berlin Definition
}

\section{OPEN ACCESS}

Edited by:

Claire Joanne Stocker,

Aston University, United Kingdom

Reviewed by:

Nadir Yehya,

Children's Hospital of Philadelphia,

United States

Flavio Protasio Veras,

University of São Paulo, Brazil

Zhangyan Lyu,

Chinese Academy of Medical Sciences and Peking Union Medical

College, China

*Correspondence: Wei Zhang

zhangwei2384@126.com

tORCID:

Wei Zhang

orcid.org/0000-0001-5806-0469

Yadan Wang

orcid.org/0000-0001-8277-7398

Specialty section:

This article was submitted to

Obesity,

a section of the journal

Frontiers in Endocrinology

Received: 29 September 2020

Accepted: 21 December 2020

Published: 12 February 2021

Citation:

Zhang W, Wang $Y$,

Li W and Wang $J(2021)$

Association Between Obesity and Short-And Long-Term Mortality in Patients With Acute Respiratory Distress Syndrome Based on the Berlin Definition.

Front. Endocrinol. 11:611435. doi: 10.3389/fendo.2020.611435

\author{
Wei Zhang ${ }^{1 * t}$, Yadan Wang ${ }^{2 \dagger}$, Weijie $\mathrm{Li}^{1}$ and Jun Wang ${ }^{1}$ \\ ${ }^{1}$ Department of Respiratory and Critical Care Medicine, Shaanxi Provincial People's Hospital, Xi'an, China, ${ }^{2}$ Medical \\ Department, Ruibiao (Wuhan) Biotechnology Co. Ltd, Wuhan, China
}

Purpose: Acute respiratory distress syndrome (ARDS) is one of the most common causes of death in intensive care units (ICU). Previous studies have reported the potential protective effect of obesity on ARDS patients. However, these findings are inconsistent, in which less was reported on long-term prognosis and diagnosed ARDS by Berlin definition. This study aimed to investigate the relationship between obesity and shortterm and long-term mortality in patients with ARDS based on the Berlin Definition.

Methods: This is a retrospective cohort study from the Medical Information Mart for Intensive Care III (MIMIC-III) database, in which all the patients were diagnosed with ARDS according to the Berlin definition. The patients were divided into four groups according to the WHO body mass index (BMI) categories. The multivariable logistic regression and Cox regression analysis were used to investigate the relationship between BMl and short-term and long-term mortality.

Result: A total of 2,378 patients with ARDS were enrolled in our study. In-hospital mortality was $27.92 \%$, and 1,036 (43.57\%) patients had died after 1-year follow-up. After adjusting for confounders, the in-hospital and 1-year mortality risks of obese patients were significantly lower than those of normal weight (OR $0.72,95 \% \mathrm{Cl} 0.55-0.94, \mathrm{P}=0.0168$; $\mathrm{HR} 0.80,95 \% \mathrm{Cl} 0.68-0.94 \mathrm{P}=0.0084$; respectively), while those mortality risks of underweight patients were higher than normal weight patients $(P=0.0102, P=0.0184$; respectively). The smooth curve showed that $\mathrm{BMI}$, which was used as a continuous variable, was negatively correlated with in-hospital and 1-year mortality. The results were consistent after being stratified by age, gender, race, type of admission, severity of organ dysfunction, and severity of ARDS. The Kaplan-Meier survival curves showed that obese patients had significant lower 1-year mortality than normal weight patients.

Conclusion: We found that obesity was associated with decreased risk of short-term and long-term mortality in patients with ARDS.

Keywords: ARDS, obesity, body mass index, 28-day mortality, 1-year mortality 


\section{INTRODUCTION}

Acute respiratory distress syndrome (ARDS) is a fatal form of acute respiratory failure, which is caused by direct (pneumonia or aspiration) or indirect lung injuries (sepsis or trauma) (1). It is one of the most common causes of death in intensive care units (ICU) and an important public health problem (2). In a recent study which involved 29,114 patients in the ICU from 50 countries, $10 \%$ of the patients who were admitted to ICU and $23 \%$ of the patients on mechanical ventilation were diagnosed with ARDS. Approximately 35-46\% of patients with ARDS died consequently during hospitalization (3). Despite the efforts in early diagnosis and treatment, to our knowledge, there is no available treatment which can aim directly at the pathological mechanism of ARDS, and mechanical ventilation and supportive care are still the main approaches $(1,2,4)$. The mortality of ARDS is still very high. Therefore, the recognition of poor prognostic factors is helpful for clinicians to adjust treatment strategies early and ultimately improve the prognosis of patients with ARDS.

Obesity has been on the rise over the past 30 years due to the changes of our feeding habits and life Style. According to the 2017 Global Nutrition Report, 2 billion adults were obese or overweight worldwide and about one in five patients in ICU was obese (5). In recent years, some studies have shown that obesity can reduce mortality in critically ill patients (5-7), such as those with chronic heart disease, chronic renal insufficiency, and sepsis, this phenomenon is termed obesity paradox (8-11); Yet whether this phenomenon exists in ARDS is still controversial (12-20). Most of the previous studies were conducted with a limited sample size, and ARDS as defined by the Berlin standard was rarely applied. Moreover, these studies mainly focused on short-term prognosis, the long-term prognosis is unclear. Our study investigated a cohort of patients with ARDS based on the current definitions and guidelines (Berlin definition), with the primary outcomes of in-hospital and 1-year mortality, to assess the effect of obesity on mortality and the course of disease.

\section{METHODS AND MATERIALS}

\section{Data Sources}

The present study is a retrospective cohort study of 2,378 patients with ARDS according to the Berlin definition. All the data used for analysis were obtained from the Medical Information Mart for Intensive Care III (MIMIC-III), which is a single-center and freely accessible database and contains 38,597 adult patients (aged over 16) during 2001 to 2012 in the ICU of the Beth Israel Deaconess Medical Center in Boston. We were obliged to complete the online course and pass the online exams (no. 6182750) to gain access to the database. The establishment of the MIMIC III database was approved by the institutional review board of Beth Israel deacons Medical Center and Massachusetts Institute of Technology. Because all the data of health information are anonymous, the informed consent was not required.

\section{Patients}

The MIMIC-III database contains the admission and discharge diagnosis information of all patients. Combined with the diagnostic information recorded in the database and the Berlin standard, we selected the patients diagnosed as ARDS for analysis. Our inclusion criteria were as follows: (1) Patients were older than 16 years and stayed in the ICU more than $48 \mathrm{~h}$; (2) Patients had an arterial oxygen partial pressure $(\mathrm{PaO} 2)$ /fraction of inspired oxygen $(\mathrm{FiO} 2)<$ $300 \mathrm{mmHg}$ and positive end-expiratory pressure $(\mathrm{PEEP}) \geq 5 \mathrm{~cm}$ $\mathrm{H}_{2} \mathrm{O}$ on the first day of ICU admission; (3) Patients with acute onset were assumed to have recently used mechanical ventilation during ICU stay; (4) Patients whose chest radiographs showed bilateral infiltration, and absence of heart failure. Patients lacking of height or weight were excluded. If a patient was admitted repeatedly during the study period, we used only the record of his/her first ICU admission.

The patients' BMI was calculated as weight $(\mathrm{Kg}) /$ height $^{2}(\mathrm{~m})$. According to the BMI international classification of World Health Organization(WHO), we divided the study population into four groups: underweight (BMI $<18.5 \mathrm{~kg} / \mathrm{m}^{2}$ ), normal weight (BMI $\geq 18.5,<25 \mathrm{~kg} / \mathrm{m}^{2}$ ), overweight (BMI $\geq 25,<30$ $\left.\mathrm{kg} / \mathrm{m}^{2}\right)$ and obese $\left(\mathrm{BMI} \geq 30 \mathrm{~kg} / \mathrm{m}^{2}\right)$.

According to the Berlin Definition, ARDS were classified into mild (> $200 \mathrm{mmHg}, \leq 300 \mathrm{mmHg}$ ), moderate $(>100 \mathrm{mmHg}, \leq$ $200 \mathrm{mmHg})$, and severe $(<100 \mathrm{mmHg})$ based on the $\mathrm{PaO} 2 /$ $\mathrm{FiO} 2$ ratio.

\section{Variables and Outcome Measures}

We used the Structured Query Language to extract the data and the cords of the Structured Query Language were obtained from https://github.com/MIT-LCP/mimic-website. We extracted or calculated the following variables, including the baseline characteristics (age, gender, ethnicity, admission type, ICU type), the patients' comorbidity, the vital signs within $24 \mathrm{~h}$ after ICU admission (heart rate, temperature, mean arterial pressure and $\mathrm{SPO}_{2}$ ), severity of organ dysfunction (Simplified Acute Physiology Score, SAPSII; Oxford Acute Severity of Illness Score, OASIS; Sequential Organ Failure Assessment, SOFA) and the characteristics of mechanical ventilation on the first day. If a variable was measured multiple times during the study period, we took its average value. The serious scores of organ dysfunction was estimated for all patients within $24 \mathrm{~h}$ of ICU admission.

The primary outcomes were the in-hospital and 1-year mortality following ICU admission, and the secondary outcomes were ICU mortality, ICU length of stay, and inhospital length of stay.

\section{Statistical Analysis}

We used the EmpowerStats software (www.empowerstats.com version R.3.4.3) and statistical software package $\mathrm{R}$ to process and analyze all our data. The categorical variables were presented as percentages and the continuous variables were expressed as the mean (SD) or IQR in the tables in our study. All the variables were compared by student t-test (normal distribution) or Mann-Whitney (non-normal distribution) when they were categorical variables, and 
by Kruskal-Wallis test when they were continuous variables. A twotailed $\mathrm{P}$ value $<0.05$ was considered statistically significant.

Multivariable logistic regression analysis and smooth curve fitting were performed to test the independent effects of obesity on ICU and in-hospital mortality with crude and full models. Multivariable Cox regression models and generalized linear models with a logit link were used to test the independent effects of obesity on 1-year all-cause mortality. The adjusting variables included age, gender, ethnicity, admission type, ICU type, sepsis, chronic pulmonary disease, renal failure, liver disease, metastatic cancer, chronic heart disease, cerebrovascular disease, Elixhauser comorbidity score, vital signs within $24 \mathrm{~h}$ after ICU admission, arterial $\mathrm{pH}$, lactic acid and the support treatment. Additionally, the log-rank test in the Kaplan-Meter survival analysis was used to compare the different survival rates between each group. Furthermore, a subgroup analysis was performed to determine whether there were differences among each subgroup in BMI prediction of clinical outcomes.

\section{RESULTS}

\section{Characteristics and Outcomes of Patients}

The flowchart of study cohort selection was shown in Figure 1. A total of 2,378 patients with ARDS were included in this study, containing 107 underweight patients, 710 normal weight patients, 749 overweight patients, and 812 obese patients. Table 1 displayed the characteristics of the study participants and Table 2 displayed the clinical outcomes of the subjects across the BMI strata.

The average age of the study participants was $61.90 \pm 17.48$ years and $59.92 \%$ of participants were male. In total, 664 participants $(27.92 \%)$ and 1,036 participants $(43.57 \%)$ died in hospital and within one year, respectively. Obese patients tended to be younger and obese female patients were more than normal weight patients. In obese patients, the incidence of common comorbidities such as diabetes and chronic lung disease is significantly higher. The SAPSII and OASIS scores within 24hour of ICU admission were significantly lower in obese patients than those in normal weight patients. These imbalanced variables may be confounding and were adjusted in subsequent analyses.

Furthermore, obese patients needed higher PEEP, plateau pressure, minute ventilation, and $\mathrm{FiO} 2$ than normal weight patients. And they had a lower ICU in-hospital and 1-year mortality than normal weight patients. There was no difference on ICU length of stay and in-hospital length of stay between obese patients and normal weight patients.

\section{Obesity and Short-Term Mortality}

The univariate logistic regression analysis for ICU and inhospital mortality was shown in Table S1. There was a significant association between patients' variables (age, ethnicity, BMI, admission type, ICU type, renal failure, liver disease, metastatic cancer, chronic heart disease, sepsis, vital signs within $24 \mathrm{~h}$ after ICU admission, arterial $\mathrm{pH}$, lactic acid, SAPSII, OASIS, SOFA, plateau pressure and FiO2) and inhospital mortality.

In multivariable logistic regression analysis, after adjusting for the clinical confounders listed, we found that obese patients had significant lower risks of the in-hospital (OR 0.72, 95\%CI 0.550.94, $\mathrm{P}=0.0168)$ and ICU (OR 0.83, 95\%CI 0.53-0.93 P=0.0140) mortality than patients with normal weights, while the underweight patients had significant higher risks of the inhospital (OR 1.87 95\%CI 1.16, $3.02 \mathrm{P}=0.0102$ ) and ICU (OR 1.76 95\%CI 1.08, $2.87 \mathrm{P}=0.0244$ Table 3) mortality.

Taking BMI as a continuous variable, we used the adjusted spline smoothing fitting to visually assess the relationship between BMI and in-hospital mortality, which were presented in Figure 2. The results showed that BMI was negatively associated with in-hospital mortality in crude and adjusted models.

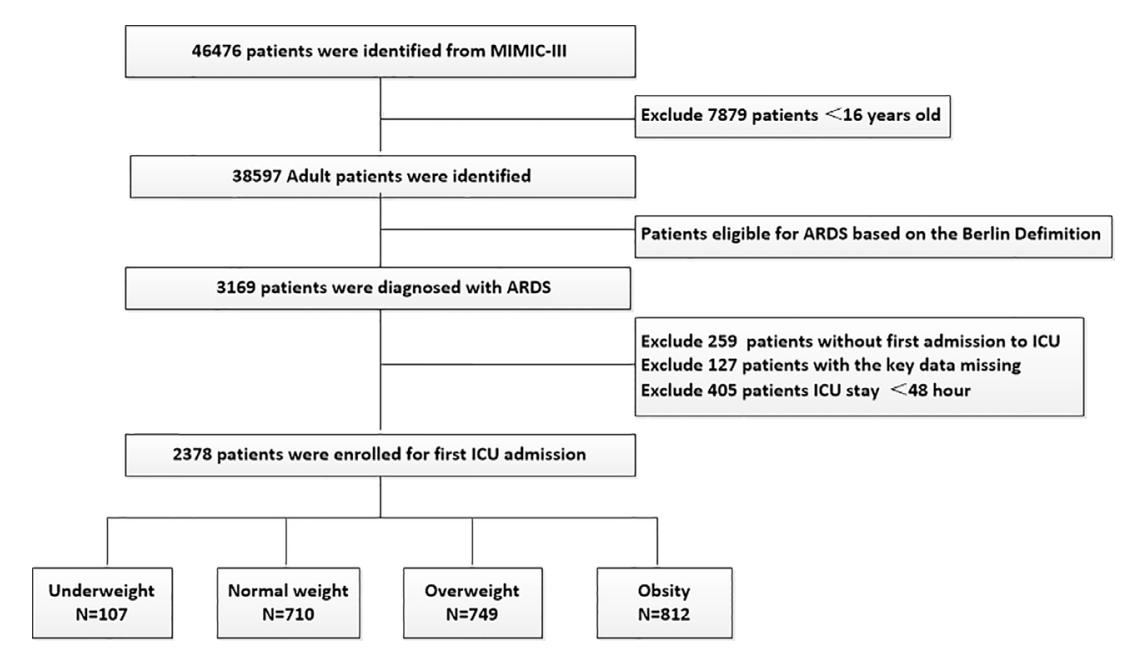

FIGURE 1 | Flow chart of the current study. 
TABLE 1 | Characteristics of the study patients according to BMI.

\begin{tabular}{|c|c|c|c|c|c|c|}
\hline Variables & All patients & Underweight <18.5 & Normal weight $\geq 18.5,<25$ & Overweight $\geq 25,<30$ & Obesity $\geq \mathbf{3 0}$ & P-value \\
\hline \multicolumn{7}{|l|}{ Baseline characteristics } \\
\hline $\mathrm{n}$ & 2378 & 107 & 710 & 749 & 812 & \\
\hline Age (years) & $61.90 \pm 17.48$ & $67.22 \pm 16.72$ & $64.10 \pm 19.22$ & $62.09 \pm 17.58$ & $59.12 \pm 15.33$ & $<0.001$ \\
\hline Male & 1425 (59.92\%) & 60 (56.07\%) & 442 (62.25\%) & $488(65.15 \%)$ & 435 (53.57\%) & $<0.001$ \\
\hline \multicolumn{7}{|l|}{ Ethnicity } \\
\hline Caucasian & 1647 (69.26\%) & 69 (64.49\%) & 482 (67.89\%) & 525 (70.09\%) & $571(70.32 \%)$ & 0.200 \\
\hline Black & $522(21.95 \%)$ & 27 (25.23\%) & $161(22.68 \%)$ & $173(23.10 \%)$ & $161(19.83 \%)$ & \\
\hline Others & $209(8.79 \%)$ & $11(10.28 \%)$ & 67 (9.44\%) & $51(6.81 \%)$ & 80 (9.85\%) & \\
\hline \multicolumn{7}{|l|}{ Admission type } \\
\hline Elective & 335 (14.09\%) & $13(12.15 \%)$ & 96 (13.52\%) & 96 (12.82\%) & $130(16.01 \%)$ & 0.262 \\
\hline Emergency/Urgent & $2043(85.91 \%)$ & $94(87.85 \%)$ & $614(86.48 \%)$ & $653(87.18 \%)$ & $682(83.99 \%)$ & \\
\hline \multicolumn{7}{|l|}{ ICU type } \\
\hline CCU/CSRU & 236 (9.92\%) & 8 (7.48\%) & 59 (8.31\%) & 79 (10.55\%) & 90 (11.08\%) & 0.059 \\
\hline $\mathrm{MICU}$ & 1251 (52.61\%) & $68(63.55 \%)$ & 367 (51.69\%) & 379 (50.60\%) & 437 (53.82\%) & \\
\hline SICU/TSICU & $891(37.47 \%)$ & 31 (28.97\%) & 284 (40.00\%) & 291 (38.85\%) & 285 (35.10\%) & \\
\hline \multicolumn{7}{|l|}{ Comorbidity } \\
\hline Chronic pulmonary disease & 624 (26.24\%) & 38 (35.51\%) & $176(24.79 \%)$ & 167 (22.30\%) & 243 (29.93\%) & $<0.001$ \\
\hline Renal failure & $129(5.42 \%)$ & $12(11.21 \%)$ & 97 (13.66\%) & $124(16.56 \%)$ & $112(13.79 \%)$ & 0.240 \\
\hline Liver disease & 252 (10.60\%) & $11(10.28 \%)$ & $61(8.59 \%)$ & $86(11.48 \%)$ & $94(11.58 \%)$ & 0.215 \\
\hline Metastatic cancer & $156(6.56 \%$ & $11(10.28 \%)$ & $54(7.61 \%)$ & 50 (6.68\%) & 41 (5.05\%) & $<0.081$ \\
\hline Chronic heart disease & 927 (38.98\%) & 40 (37.38\%) & 301 (42.39\%) & 271 (36.18\%) & 315 (38.79\%) & 0.108 \\
\hline Cerebrovascular disease & 276 (11.61\%) & $14(13.08 \%)$ & 77 (10.85\%) & 90 (12.02\%) & 95 (11.70\%) & 0.859 \\
\hline Diabetes & $626(26.32 \%)$ & $13(12.15 \%)$ & 127 (17.89\%) & 179 (23.90\%) & 307 (37.81\%) & $<0.001$ \\
\hline Sepsis & $1322(55.59 \%)$ & $60(56.07 \%)$ & $381(53.66 \%)$ & $412(55.01 \%)$ & $469(57.76 \%)$ & 0.435 \\
\hline Elixhauser comorbidity score & $8.50 \pm 7.85$ & $10.76 \pm 8.74$ & $8.73 \pm 7.76$ & $8.56 \pm 7.70$ & $7.95 \pm 7.90$ & 0.004 \\
\hline \multicolumn{7}{|c|}{ Vital signs within $24 \mathrm{~h}$ after ICU admission } \\
\hline Heart rate $(\mathrm{bpm})$ & $90.89 \pm 16.80$ & $91.65 \pm 17.09$ & $91.21 \pm 16.78$ & $90.68 \pm 16.16$ & $90.70 \pm 17.37$ & 0.874 \\
\hline $\mathrm{MAP}(\mathrm{mmHg})$ & $76.16 \pm 9.94$ & $73.67 \pm 8.90$ & $76.00 \pm 10.15$ & $75.44 \pm 9.49$ & $77.30 \pm 10.17$ & $<0.001$ \\
\hline Temperature $\left({ }^{\circ} \mathrm{C}\right)$ & $37.08 \pm 0.77$ & $36.75 \pm 0.77$ & $37.02 \pm 0.76$ & $37.10 \pm 0.76$ & $37.15 \pm 0.78$ & $<0.001$ \\
\hline $\mathrm{SPO}_{2}$ & $96.81 \pm 3.85$ & $96.22 \pm 6.59$ & $96.96 \pm 3.17$ & $96.80 \pm 4.17$ & $96.76 \pm 3.58$ & 0.287 \\
\hline \multicolumn{7}{|l|}{ Severity of organ dysfunction } \\
\hline SAPSII & $44.51 \pm 14.76$ & $46.78 \pm 14.38$ & $45.47 \pm 14.31$ & $44.56 \pm 14.76$ & $43.31 \pm 15.10$ & 0.012 \\
\hline OASIS & $38.05 \pm 8.26$ & $39.73 \pm 8.17$ & $38.45 \pm 7.87$ & $37.95 \pm 8.44$ & $37.56 \pm 8.39$ & 0.028 \\
\hline SOFA & $7.03 \pm 3.55$ & $6.35 \pm 3.46$ & $6.92 \pm 3.56$ & $7.05 \pm 3.45$ & $7.21 \pm 3.64$ & 0.077 \\
\hline \multicolumn{7}{|c|}{ Laboratory data on the first day after ICU admission } \\
\hline Arterial $\mathrm{pH}$ & $7.36 \pm 0.08$ & $7.34 \pm 0.09$ & $7.36 \pm 0.08$ & $7.36 \pm 0.07$ & $7.35 \pm 0.08$ & 0.123 \\
\hline $\mathrm{PaCO} 2(\mathrm{~mm} \mathrm{Hg})$ & $42.40 \pm 9.70$ & $42.50 \pm 10.06$ & $41.58 \pm 9.91$ & $41.67 \pm 8.73$ & $43.80 \pm 10.18$ & $<0.001$ \\
\hline Lactic acid & $2.00 \pm 2.20$ & $1.61 \pm 1.80$ & $1.95 \pm 2.16$ & $2.15 \pm 2.43$ & $1.94 \pm 2.05$ & 0.057 \\
\hline $\mathrm{PaO} 2 / \mathrm{FiO} 2$ (mmHg) & $142.50 \pm 65.73$ & $160.80 \pm 70.42$ & $142.87 \pm 67.77$ & $140.13 \pm 64.08$ & $141.95 \pm 64.50$ & 0.025 \\
\hline \multicolumn{7}{|c|}{ Need of support in the first $24 \mathrm{~h}$} \\
\hline Renal replacement therapy & $129(5.42 \%)$ & $4(3.74 \%)$ & $43(6.06 \%)$ & 38 (5.07\%) & $44(5.42 \%)$ & 0.723 \\
\hline Vasopressor & $1212(50.97 \%)$ & $51(47.66 \%)$ & 357 (50.28\%) & 391 (52.20\%) & $413(50.86 \%)$ & 0.786 \\
\hline \multicolumn{7}{|c|}{ Characteristics of mechanical ventilation on the first day after ICU admission } \\
\hline Tidal volume (ml/kg PBW) & $9.08 \pm 3.74$ & $8.34 \pm 3.71$ & $8.62 \pm 2.30$ & $8.92 \pm 2.23$ & $9.73 \pm 5.41$ & $<0.001$ \\
\hline PEEP $(\mathrm{cmH} 2 \mathrm{O})$ & $7.44 \pm 3.36$ & $6.32 \pm 2.52$ & $7.16 \pm 2.98$ & $7.30 \pm 3.30$ & $7.95 \pm 3.73$ & $<0.001$ \\
\hline Plateau pressure (cmH2O) & $22.66 \pm 6.02$ & $20.23 \pm 5.89$ & $21.80 \pm 6.43$ & $22.55 \pm 5.73$ & $23.82 \pm 5.69$ & $<0.001$ \\
\hline Minute ventilation ( $(1 / \mathrm{min})$ & $9.10 \pm 5.72$ & $8.34 \pm 7.67$ & $8.82 \pm 6.71$ & $8.98 \pm 3.72$ & $9.56 \pm 5.98$ & 0.027 \\
\hline $\mathrm{FiO} 2(\%)$ & $60.54 \pm 12.67$ & $56.95 \pm 14.64$ & $59.68 \pm 12.30$ & $60.82 \pm 12.45$ & $61.49 \pm 12.81$ & $<0.001$ \\
\hline
\end{tabular}

BMI, Body Mass Index; ICU, intensive care units; CCU, coronary care unit; CSRU, cardiac surgery recovery unit; MICU, medical intensive care unit; SICU, surgical intensive care unit; TSICU, thoracic surgery Intensive care unit; MAP, Mean Arterial Pressure; PaO2/FiO2, arterial oxygen partial pressure/fraction of inspired oxygen; SAPSII, Simplified Acute Physiology Score; OASIS, Oxford Acute Severity of IIIness Score; SOFA, Sequential Organ Failure Assessment; PBW, predicted body weight; PEEP, positive end-expiratory pressure; bpm, breaths per minute.

TABLE 2 | Outcomes of subjects across the BMl strata.

\begin{tabular}{|c|c|c|c|c|c|c|}
\hline Variables & All patients & underweight $<18.5$ & Normal weight $\geq 18.5,<25$ & Overweight $\geq 25,<30$ & Obesity $\geq 30$ & P-value \\
\hline $\mathrm{n}$ & 2378 & 107 & 710 & 749 & 812 & \\
\hline Time in ICU (days) & $13.10 \pm 12.14$ & $11.48 \pm 10.09$ & $12.5 \pm 12.45$ & $13.7 \pm 12.78$ & $13.2 \pm 11.45$ & 0.124 \\
\hline Time in hospital (days) & $22.58 \pm 18.83$ & $21.00 \pm 14.23$ & $21.64 \pm 17.75$ & $23.35 \pm 20.35$ & $22.89 \pm 18.80$ & 0.261 \\
\hline ICU mortality & $556(23.38 \%)$ & 42 (39.25\%) & 185 (26.06\%) & $184(24.57 \%)$ & 145 (17.86\%) & $<0.001$ \\
\hline In-hospital mortality & 664 (27.92\%) & 50 (46.73\%) & 217 (30.56\%) & $221(29.51 \%)$ & 176 (21.67\%) & $<0.001$ \\
\hline 1-year mortality & 1036 (43.57\%) & 72 (67.29\%) & 351 (49.44\%) & $332(44.33 \%)$ & 281 (34.61\%) & $<0.001$ \\
\hline
\end{tabular}


TABLE 3 | Multivariate logistics regression analysis for ICU and in-hospital mortality in BMl groups.

\begin{tabular}{|c|c|c|c|}
\hline BMI group & Non-adjusted & Adjust I & Adjust II \\
\hline ICU mortality & OR 95\% Cl p-value & OR 95\% Cl p-value & OR 95\% Cl p-value \\
\hline Normal weight & 1.0 (reference) & 1.0 (reference) & 1.0 (reference) \\
\hline Underweight & $1.83(1.20,2.80) 0.0049$ & $1.73(1.12,2.66) 0.0135$ & $1.76(1.08,2.87) 0.0244$ \\
\hline Overweight & $0.92(0.73,1.17) 0.5128$ & $0.97(0.76,1.23) 0.7925$ & $0.93(0.71,1.22) 0.5952$ \\
\hline Obesity & $0.62(0.48,0.79) 0.0001$ & $0.70(0.54,0.90) 0.0049$ & $0.70(0.53,0.93) 0.0140$ \\
\hline$p$ for trend & $<0.0001$ & 0.0047 & 0.0145 \\
\hline In-hospital mortality & OR 95\% Cl p-value & OR 95\% Cl p-value & OR 95\% Cl p-value \\
\hline Normal weight & 1.0 (reference) & 1.0 (reference) & 1.0 (reference) \\
\hline Underweight & $1.99(1.32,3.01) 0.0010$ & $1.89(1.24,2.88) 0.0032$ & $1.87(1.16,3.02) 0.0102$ \\
\hline Overweight & $0.95(0.76,1.19) 0.6596$ & $0.99(0.79,1.25) 0.9590$ & $0.97(0.75,1.25) 0.7866$ \\
\hline Obesity & $0.63(0.50,0.79)<0.0001$ & $0.71(0.56,0.90) 0.0052$ & $0.72(0.55,0.94) 0.0168$ \\
\hline$p$ for trend & $<0.0001$ & 0.0048 & 0.0186 \\
\hline
\end{tabular}

Adjusted I for age, gender and ethnicity;

Adjusted II for age, gender, ethnicity, admission type, ICU type, sepsis, chronic pulmonary disease, renal failure, liver disease, metastatic cancer, chronic heart disease, cerebrovascular disease, Elixhauser comorbidity score, vital signs within 24h after ICU admission, SAPSII, SOFA, OASIS, arterial pH, lactic acid, renal replacement therapy and vasopressor.

\section{Obesity and Long-Term Mortality}

In univariate COX analysis (Table S2), BMI showed a strong relation with long-term survival as illustrated by the KaplanMeier curves (log-rank test $\mathrm{P}<0.001$, Figure 3). In the multivariable Cox regression analysis, obese patients had significant lower risks of 1-year mortality (HR 0.63, 95\%CI 0.53-0.73 $\mathrm{P}<0.0001)$ than patients with normal weights. After adjusting for potential confounders, the association remained independent (HR 0.80, 95\% CI 0.68-0.94 $\mathrm{P}=0.0084$, Table 4, model 2). Moreover we used the generalized additive models to visually assess the relationship between BMI and 1-year mortality (Figure 4). The result was similar with the shortterm outcomes.

\section{Subgroup Analyses}

The subgroup analyses for the relationship between BMI and the in-hospital and 1-year mortality were presented in Tables $\mathbf{5}$ and 6, which were performed according to age, gender, race, type of admission, severity of ARDS, SAPSII, OASIS and SOFA scores. The results showed that in different subgroups, the relationship between BMI and the risk of in-hospital and 1-year mortality stably existed after careful adjustments. The association between obesity and long-term mortality was only not observed in other race patients (OR 1.03, 95\% CI 0.53-1.99, $\mathrm{P}=0.9269$ ).

\section{DISCUSSION}

The results of this study suggested that there was an independent association between BMI and the short-term and long-term mortality of ARDS patients according to the Berlin standard. Underweight patients had higher risks of short-term and longterm mortality, while obese patients had lower risks of mortality compared with normal weight patients. In addition, according to the Berlin standard, patients were divided into three groups (mild, moderate, and severe ARDS) based on their $\mathrm{PaO} 2 / \mathrm{FiO} 2$ ratio with the first record after ICU admission. Obesity was also found to be associated with a decreased risk of short-term and long-term mortality in ARDS patients despite of the severity.
As far as we know, there were some previous studies about the relationship between BMI and the mortality of ARDS, but their conclusions were controversial $(12,15-20)$. Most of these studies had shown that BMI was inversely associated with the mortality of the patients with acute lung injury (ALI) or ARDS, and obesity or morbidly obesity could reduce the risk of mortality, which was consistent with our finding $(13,14,16-20)$. Other studies suggested that there was no association between BMI and the mortality of ARDS $(12,15)$. In addition, there were two meta-analyses on BMI and the clinical prognosis of ARDS. Yue et al. reported that in a meta-analysis which included 6,268 patients with ARDS or ALI, the OR of ICU mortality was $0.68(95 \%$ CI $0.57,0.80)$ for obese patients and $0.72(95 \%$ CI $0.56,0.93)$ for morbidly obese patients respectively (13). Another meta-analysis which included 9 studies showed that obesity significantly decreased the risk of 60-day and 90-day mortality, OR values was 0.84 (95\% CI 0.75 to -0.94$)$ and 0.38 (95\% CI 0.22-0.66) (14). These inconsistent results may be due to the following reasons. First, in different investigations, there were different cut-off values of BMI, different ages, and different races of individuals; Second, the study population was included according to different diagnostic criteria for ARDS and ALI and most of these studies used the American European Consensus Conference definitions; Third, there were significant differences in some studies due to the lack of adjustments for potential important confounding factors, including comorbid illness (such as diabetes mellitus and acute renal insufficiency, which had been shown to be associated with the onset and prognosis of ARDS $(16,21)$ ) and disease severity (APS III score, SIRS score and the value of oxygenation index).

In the present study, we selected patients according to the Berlin definition for ARDS, which is the current definition and guidelines, and we also defined obesity according to WHO standards. We had adjusted for important comorbid illnesses (including Elixhauser Comorbidity index) and disease severity (including SAPSII, OASIS, SOFA, and the value of oxygenation index) because MIMIC-III database had complete patients' records. In addition, we had investigated the association between BMI and 1-year mortality of ARDS. To our knowledge, this association has not been reported in previous studies. 
A

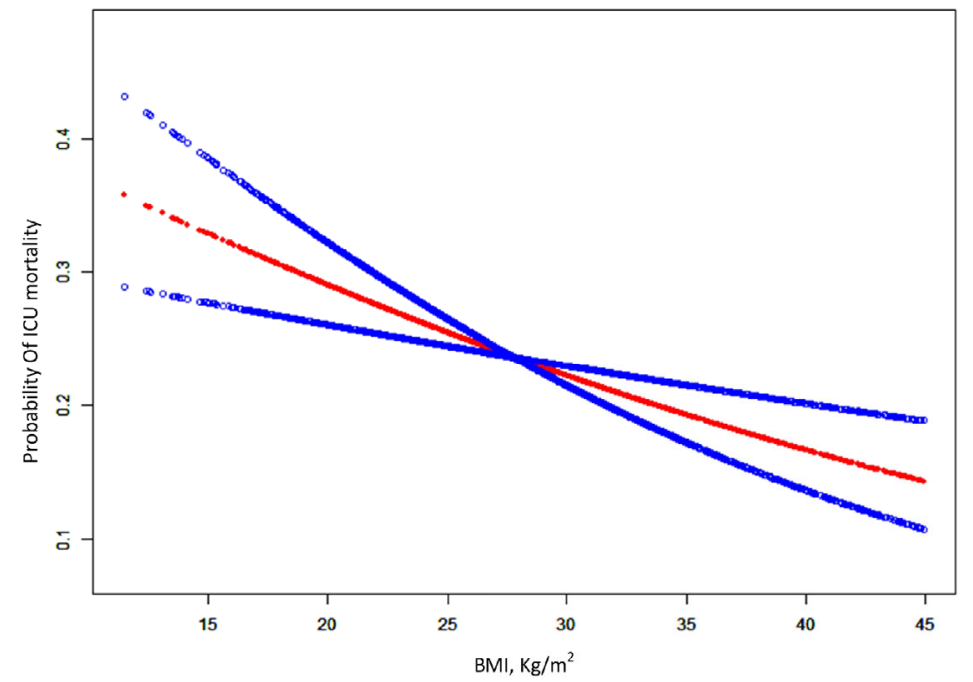

B

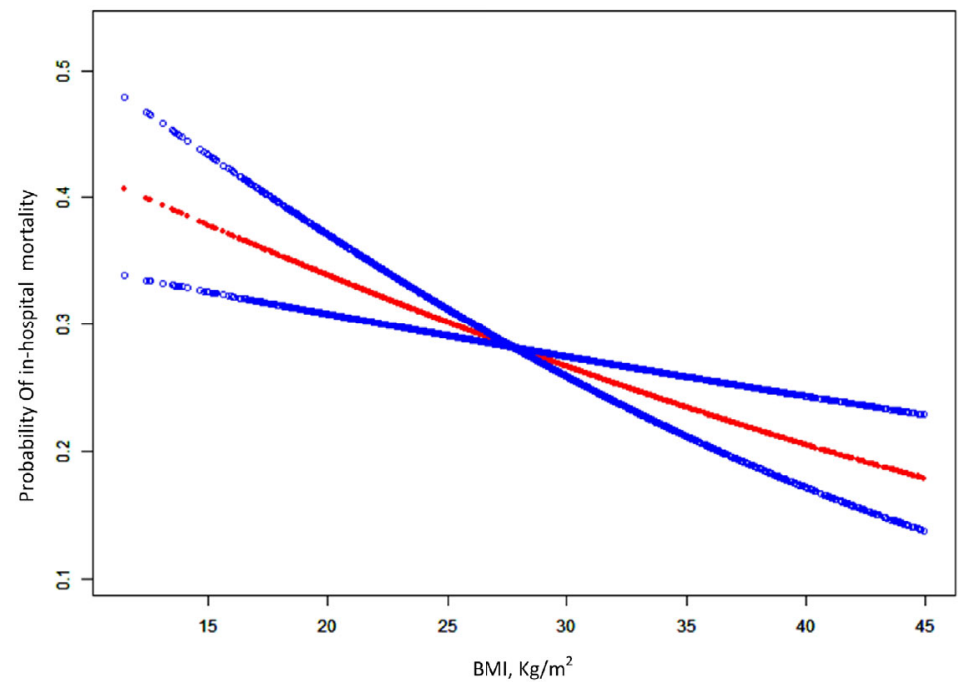

FIGURE 2 | A smooth curve fitting for the relationship between BMI and the risk of ICU and in-hospital mortality. (A) Association between BMI and ICU mortality for patients with ARDS. (B) association between BMl and in-hospital for patients with ARDS. The resulting figures show the risk of mortality in the $y$-axis and the BMl (continuous variable) in the $\mathrm{x}$-axis. A negative relationship between $\mathrm{BMl}$ and the risk of short-term mortality was observed after adjusting for age, gender, ethnicity, admission type, ICU type, sepsis, chronic pulmonary disease, renal failure, liver disease, metastatic cancer, chronic heart disease, cerebrovascular disease,

Elixhauser comorbidity score, vital signs within 24h after ICU admission, SAPSII, SOFA, OASIS, arterial pH, lactic acid, renal replacement therapy and vasopressor by spline smoothing plot.

Our study had a large sample size and adjusted for multiple confounders, which were improvements on previous studies. As a retrospective observational study, we found an association between obesity and a decreased risk of death, however we were unable to establish a causal relationship between obesity and mortality. In fact, ARDS may be over-diagnosed in obese subjects, as the chest wall can more readily induce atelectasis masquerading as bilateral infiltrates on chest $\mathrm{x}$-ray. This leads to (easily reversible) hypoxemia, which makes the subject appear more sick than they really are. On the other hand, due to the existence of collider bias, the real association between obesity and mortality is artificially biased forward being falsely "protective" by conditioning on the diagnosis of ARDS $(22,23)$. Therefor based on our data, obesity was associated with a decreased risk of mortality for ARDS patients. Due to the collider bias and the possibility that obese patients may be over-diagnosed with ARDS, it was unclear whether obesity paradox existed in these patients. 


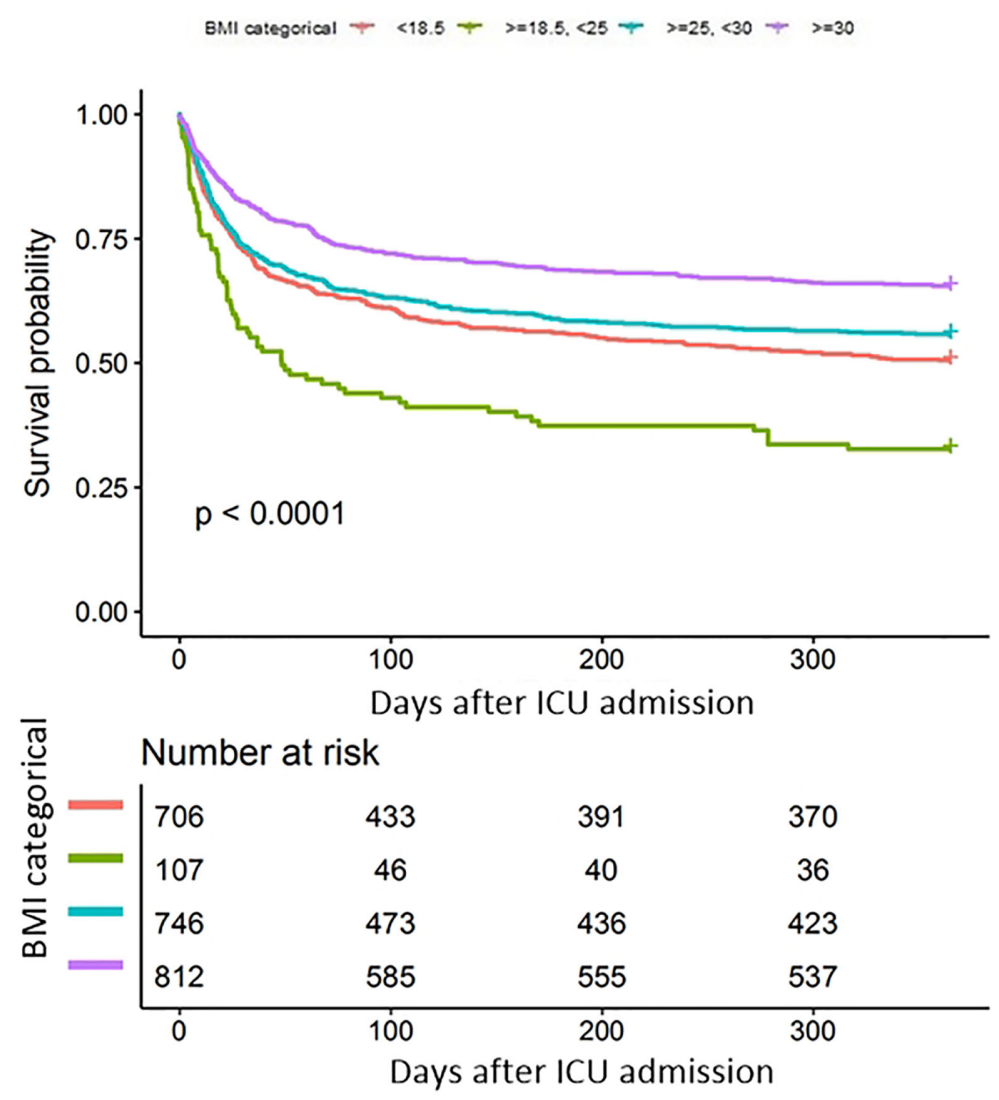

FIGURE 3 | Kaplan-Meier (K-M) survival curves of 1-year mortalities by BMI categorical.

TABLE 4 | Multivariable Cox regression analysis for 1-year mortality in BMl groups

\begin{tabular}{|c|c|c|c|}
\hline BMI group & Non-adjusted & Adjust I & Adjust II \\
\hline 1-year mortality & HR 95\% Cl p-value & HR 95\% Cl p-value & HR 95\% Cl p-value \\
\hline Normal weight & 1.0 (reference) & 1.0 (reference) & 1.0 (reference) \\
\hline Underweight & $1.66(1.29,2.14)<0.0001$ & $1.54(1.20,1.99) 0.0008$ & $1.37(1.05,1.78) 0.0184$ \\
\hline Overweight & $0.87(0.75,1.01) 0.0765$ & $0.93(0.80,1.09) 0.3683$ & $0.92(0.79,1.08) 0.3101$ \\
\hline Obesity & $0.63(0.53,0.73)<0.0001$ & $0.73(0.63,0.86) 0.0002$ & $0.80(0.68,0.94) 0.0084$ \\
\hline$p$ for trend & $<0.0001$ & $<0.0001$ & 0.0061 \\
\hline
\end{tabular}

Adjusted I for age, gender and ethnicity;

Adjusted II for age, gender, ethnicity, admission type, ICU type, sepsis, chronic pulmonary disease, renal failure, liver disease, metastatic cancer, chronic heart disease, cerebrovascular disease, Elixhauser comorbidity score, vital signs within $24 \mathrm{~h}$ after ICU admission, SAPSII, SOFA, OASIS, arterial pH, lactic acid, renal replacement therapy and vasopressor

The physiologic mechanisms behind these associations are not yet clear. We can give some possible reasons why obesity can improve clinical prognosis of ARDS patients. First, obesity is a medical condition with pro-inflammatory components and obese patients have higher cytokines in the peripheral circulation which may induce antioxidant consumption and endothelial damage leading to increased capillary permeability and lung damage (24). However, in animal studies, obesity can lead to impaired neutrophil functions (including cytokines transcription and downstream signaling pathways), the transformation of alveolar macrophages into an antiinflammatory phenotype, and a reduction in the number of monocytes and adhesion receptors compared with normal weight individuals $(25,26)$. In addition, previous studies have shown that pro-inflammatory factors in the peripheral circulation of obese ARDS patients are not significantly higher than those of normal weight patients (27). Furthermore, in animal with acute lung injury, it was found that the phenotypes of neutrophils and monocytes/macrophages in BALF and blood of obese rats were changed, making them 


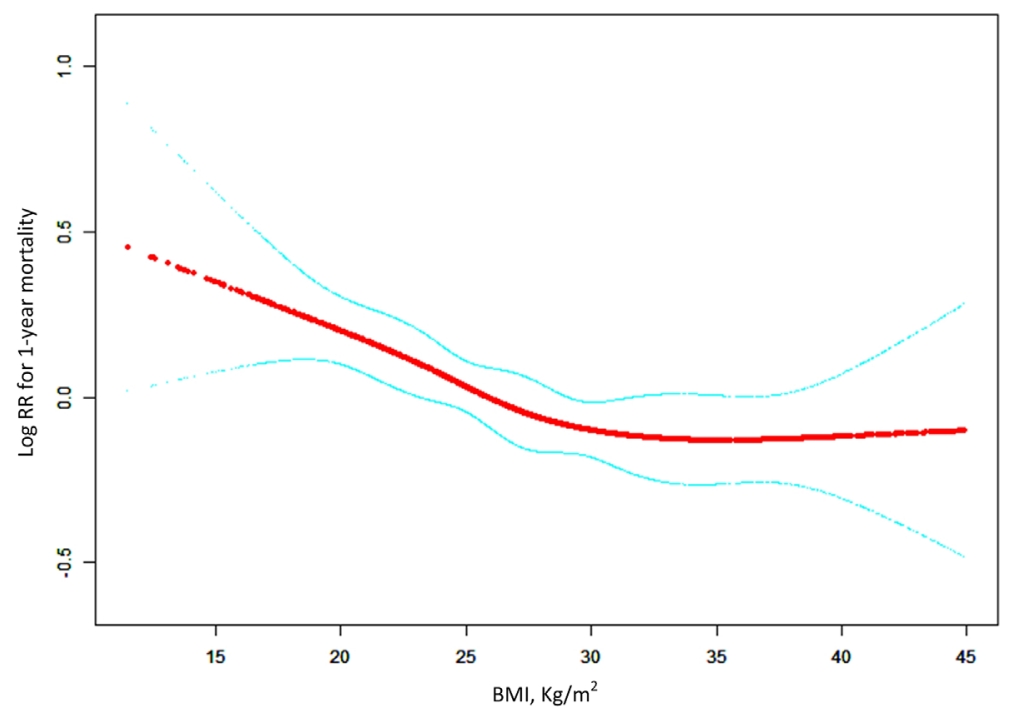

FIGURE 4 | General additive models demonstrate the relationship between BMI and the risk of 1-year mortality in ARDS patients. The resulting figures show the predicted log(relative risk) in the $y$-axis and the BMI in the x-axis. The model was adjusted for age, gender, ethnicity, admission type, ICU type, sepsis, chronic pulmonary disease, renal failure, liver disease, metastatic cancer, chronic heart disease, cerebrovascular disease, Elixhauser comorbidity score, vital signs within $24 \mathrm{~h}$ after ICU admission, SAPSII, SOFA, OASIS, arterial pH, lactic acid, renal replacement therapy and vasopressor.

produce anti-inflammatory and anti-fibrosis effects and preventing further deterioration of lung function (25). Thus the chronic inflammation of obesity may induce tolerance after an acute inflammation, low-grade inflammation may protect the lungs from further damage by preventing more severe secondary inflammation (24). Second, ARDS is a hypercatabolic state. Due to the high lipid bank, obese patients can provide more substrate synthesis energy to meet the higher demands during the initial catabolic phase of the disease (5). Obese patients can also provide more lipoproteins which can bind to endotoxins and reduce their inflammatory effects $(4,21)$. Third, some studies reported that obesity can reduce the occurrence of ventilator-induced lung injury in ARDS patients $(28,29)$. In obesity, the altered chest wall dynamic can reduce the impact of airway pressure, thereby providing protection against ventilator-induced lung injury (4). And an animal experiment showed that in obese mice with ARDS, adipose-derived exosomes can protect the lung from endothelial barrier injury and reduce the inflammatory response by inhibiting TRPV4/Ca ${ }^{2+}$ signaling pathway, thus reducing the occurrence of ventilator-related lung injury (30). Finally, clinicians generally consider that there is a higher risk of mortality for obese patients compared with normal weight patients. And this may lead to earlier admission to ICU and prompt prevention measures (including tighter blood sugar control and greater attention to ventilator parameters) which can reduce the risk of death in these patients (31).

Another finding of the present study was that obese patients had higher PEEP, plateau pressure, minute ventilation, and FiO2 than normal weight patients, which have also been described in obese patients in some previous studies. Christian et al. have found that higher PEEP can improve the survival rate of obese ARDS patients (32). In ARDS patients, high PEEP can reduce the lung injury caused by repeated collapse and reopening of the alveoli, thus improving oxygen cooperation. There was a difference in atelectasis between obese and normal patients. In obese patients, respiratory compliance decreased, lung resistance increased, and alveolar atelectasis increased. Therefore, increased PEEP may have a protective effect on obese patients to reduce the damage of repeated collapse and reopening of the alveoli to the lung, thus improving oxygen concentration. In addition, previous studies have found that obese and severely obese ARDS patients need high platform pressure and minute ventilation $(24,33)$. However, after adjusting for confounding factors, high platform pressure and minute ventilation were not significantly associated with the prognosis of patients. Therefore, we speculated that high PEEP may be another reason why obesity can reduce the risk of mortality in ARDS patients.

In the present study, we had four limitations. First, as a retrospective cohort study, it is impossible to adjust for all confounders. Because several variables were not recorded in MIMIC-III, we lacked information on the clinic risks for ARDS (including sepsis, pneumonia, trauma, and aspiration) which may affect our results. Second, we used the height and weight recorded at ICU admission to calculate BMI. However, it cannot be excluded that whether the patient underwent fluid resuscitation prior to ICU admission. Third, the results presented in this study were associations and do not imply causality, and the molecular and physiological mechanisms behind it need to be further studied and clarified. Fourth, as a single-center study, the results should be interpreted with caution when referring to other populations and regions. 
TABLE 5 | Subgroup analysis for the effect of obesity on risk of in-hospital mortality after adjusting for confounding factors.

\begin{tabular}{|c|c|c|c|c|c|}
\hline BMI & $\mathbf{N}$ & Normal weight reference & Underweight OR $95 \% \mathrm{Cl}$ p-value & Overweight OR $95 \% \mathrm{Cl} \mathrm{p}$-value & Obesity OR $95 \% \mathrm{CI}$ p-value \\
\hline$<65$ & 1266 & 1.0 & $3.44(1.59,7.45) 0.0018$ & $0.91(0.60,1.38) 0.6572$ & $0.83(0.55,1.25) 0.3771$ \\
\hline \multicolumn{6}{|l|}{ Gender } \\
\hline Male & 1425 & 1.0 & $1.88(0.93,3.81) 0.0781$ & $0.95(0.68,1.34) 0.7866$ & $0.67(0.46,0.98) 0.0368$ \\
\hline Female & 953 & 1.0 & $2.48(1.16,5.32) 0.0197$ & $0.99(0.63,1.54) 0.9574$ & $0.80(0.51,1.25) 0.3309$ \\
\hline \multicolumn{6}{|l|}{ Ethnicity } \\
\hline \multirow{2}{*}{\multicolumn{6}{|c|}{ Admission type }} \\
\hline & & & & & \\
\hline Elective & 335 & 1.0 & $2.73(0.46,16.15) 0.2672$ & $0.90(0.37,2.21) 0.8230$ & $0.84(0.34,2.10) 0.7163$ \\
\hline \multicolumn{6}{|l|}{ SAPSII } \\
\hline$<43$ & 1151 & 1.0 & $1.65(0.67,4.06) 0.2743$ & $1.35(0.87,2.08) 0.1817$ & $0.51(0.31,0.85) 0.0103$ \\
\hline \multicolumn{6}{|c|}{ 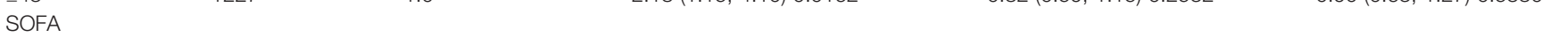 } \\
\hline \multicolumn{6}{|r|}{ 每 } \\
\hline$<38$ & 1161 & 1.0 & $3.05(1.33,7.00) 0.0086$ & $1.12(0.74,1.69) 0.5947$ & $0.58(0.36,0.94) 0.0271$ \\
\hline$\geq 38$ & 1217 & 1.0 & $1.64(0.87,3.08) 0.1239$ & $0.88(0.62,1.25) 0.4692$ & $0.86(0.60,1.23) 0.4162$ \\
\hline \multicolumn{6}{|l|}{ ARDS } \\
\hline Mild & 493 & 1.0 & $1.02(0.85,1.51) 0.2080$ & $1.05(0.57,1.93) 0.8778$ & $0.65(0.34,1.22) 0.1781$ \\
\hline Moderate & 1110 & 1.0 & $2.75(1.34,5.65) 0.0060$ & $1.04(0.70,1.55) 0.8423$ & $0.72(0.46,1.10) 0.1313$ \\
\hline Severe & 775 & 1.0 & $2.03(0.81,5.05) 0.1297$ & $0.87(0.57,1.33) 0.5068$ & $0.79(0.51,1.23) 0.2973$ \\
\hline
\end{tabular}

TABLE 6 | Subgroup analysis for the effect of obesity on risk of 1-year mortality after adjusting for confounding factors.

\begin{tabular}{|c|c|c|c|c|c|}
\hline BMI & $\mathbf{N}$ & Normal weight reference & Underweight $\mathrm{HR} 95 \% \mathrm{Cl}$ p-value & Overweight $\mathrm{HR} 95 \% \mathrm{Cl}$ p-value & Obesity HR $95 \% \mathrm{Cl} \mathrm{p}$-value \\
\hline$<65$ & 1266 & 1.0 & $2.09(1.36,3.22) 0.0007$ & $0.94(0.72,1.22) 0.6283$ & $0.85(0.65,1.10) 0.2182$ \\
\hline \multicolumn{6}{|l|}{ Gender } \\
\hline Male & 1425 & 1.0 & $1.50(1.05,2.16) 0.0272$ & $0.89(0.73,1.09) 0.2565$ & $0.78(0.62,0.98) 0.0291$ \\
\hline Female & 953 & 1.0 & $1.24(0.83,1.85) 0.3006$ & $0.95(0.73,1.22) 0.6696$ & $0.82(0.63,1.05) 0.1179$ \\
\hline \multicolumn{6}{|l|}{ Ethnicity } \\
\hline other & 209 & 1.0 & $2.60(1.06,6.41) 0.0374$ & $1.40(0.71,2.77) 0.3341$ & $1.03(0.53,1.99) 0.9269$ \\
\hline \multicolumn{6}{|l|}{ Admission type } \\
\hline Elective & 335 & 1.0 & $1.48(0.48,4.54) 0.4964$ & $1.06(0.65,1.73) 0.8187$ & $0.85(0.52,1.40) 0.5330$ \\
\hline \multicolumn{6}{|l|}{ SAPSII } \\
\hline$<43$ & 1151 & 1.0 & $1.22(0.74,2.01) 0.4393$ & $1.00(0.76,1.31) 0.9961$ & $0.62(0.46,0.84) 0.0023$ \\
\hline \multicolumn{6}{|l|}{ OASIS } \\
\hline$<38$ & 1161 & 1.0 & $1.45(0.93,2.26) 0.0988$ & $0.90(0.71,1.15) 0.4074$ & $0.63(0.48,0.83) 0.0010$ \\
\hline$\geq 38$ & 1217 & 1.0 & $1.42(1.01,1.98) 0.0416$ & $0.94(0.77,1.15) 0.5501$ & $0.94(0.76,1.16) 0.5799$ \\
\hline \multicolumn{6}{|l|}{ ARDS } \\
\hline Mild & 493 & 1.0 & $1.15(0.64,2.07) 0.6363$ & $0.94(0.64,1.36) 0.7257$ & $0.74(0.50,1.09) 0.1295$ \\
\hline Moderate & 1110 & 1.0 & $1.20(0.81,1.78) 0.3596$ & $0.88(0.70,1.10) 0.2561$ & $0.75(0.58,0.97) 0.0255$ \\
\hline Severe & 775 & 1.0 & $1.39(0.86,2.24) 0.1809$ & $0.97(0.75,1.25) 0.7933$ & $0.83(0.62,1.09) 0.1812$ \\
\hline
\end{tabular}

\section{CONCLUSION}

In conclusion, our investigation indicates that BMI was independently associated with short-term and long-term mortality of ARDS patients according to the Berlin standard. Compared with normal weight, obesity had lower risk of mortality for patients with ARDS. The relationship needs to be further validated in other populations, and the mechanisms behind these associations need to 
be further investigated. Understanding how obesity decreased the risk of mortality in ARDS may affect the treatment strategies and clinical outcomes for many patients.

\section{DATA AVAILABILITY STATEMENT}

The original contributions presented in the study are included in the article/Supplementary Material. Further inquiries can be directed to the corresponding author.

\section{AUTHOR CONTRIBUTIONS}

WZ designed the study, collected, and analyzed data, and contributed to writing this manuscript. YW collected and analyzed data. JW and WL designed and supervised the study and drafted the manuscript. All authors contributed to the article and approved the submitted version.

\section{REFERENCES}

1. Fan E, Brodie D, Slutsky AS. Acute Respiratory Distress Syndrome: Advances in Diagnosis and Treatment. JAMA J Am Med Assoc (2018) 319(7):698-710. doi: 10.1001/jama.2017.21907

2. Thompson BT, Chambers RC, Liu KD. Acute Respiratory Distress Syndrome N Engl J Med (2017) 377(6):562-72. doi: 10.1056/NEJMra1608077

3. Bellani G, Laffey JG, Pham T, Fan E, Brochard L, Esteban A, et al. Epidemiology, Patterns of Care, and Mortality for Patients With Acute Respiratory Distress Syndrome in Intensive Care Units in 50 Countries. JAMA (2016) 315(8):788-800. doi: 10.1001/jama.2016.0291

4. Umbrello M, Fumagalli J, Pesenti A, Chiumello D. Pathophysiology and Management of Acute Respiratory Distress Syndrome in Obese Patients. Semin Resp Crit Care (2019) 40(1):40. doi: 10.1055/s-0039-1685179

5. Karampela I, Chrysanthopoulou E, Christodoulatos GS, Dalamaga M. Is There an Obesity Paradox in Critical Illness? Epidemiologic and Metabolic Considerations. Curr Obes Rep (2020) 9(3):231-44. doi: 10.1007/s13679-020-00394-x

6. Zhou D, Li Z, Shi G, Zhou J. Obesity paradox for critically ill patients may be modified by age: a retrospective observational study from one large database. Crit Care (2020) 24(1):425. doi: 10.1186/s13054-020-03157-1

7. Endalifer ML, Diress G. Epidemiology, Predisposing Factors, Biomarkers, and Prevention Mechanism of Obesity: A Systematic Review. J Obes (2020) 2020:1-8. doi: 10.1155/2020/6134362

8. Mewes C, Bohnke C, Alexander T, Buttner B, Hinz J, Popov AF, et al. Favorable 90-Day Mortality in Obese Caucasian Patients with Septic Shock According to the Sepsis-3 Definition. J Clin Med (2019) 9(1):46. doi: 10.3390/ jcm9010046

9. Mei X, Hu S, Mi L, Zhou Y, Chen T. Body mass index and all-cause mortality in patients with percutaneous coronary intervention: A dose-response metaanalysis of obesity paradox. Obes Rev (2021) 22(2):e13107. doi: 10.1111/ obr.13107

10. Pepper DJ, Demirkale CY, Sun J, Rhee C, Fram D, Eichacker P, et al. Does Obesity Protect Against Death in Sepsis? A Retrospective Cohort Study of 55,038 Adult Patients. Crit Care Med (2019) 47(5):643-50. doi: 10.1097/ CCM.0000000000003692

11. Park J, Ahmadi SF, Streja E, Molnar MZ, Flegal KM, Gillen D, et al. Obesity paradox in end-stage kidney disease patients. Prog Cardiovasc Dis (2014) 56 (4):415-25. doi: 10.1016/j.pcad.2013.10.005

12. Tlayjeh H, Arabi YM, Ferguson ND, Zhou Q, Lamontagne F, Arroliga A, et al. Body Mass Index and Mortality in Subjects With ARDS: Post-hoc Analysis of the OSCILLATE Trial. Resp Care (2019) 64(9):1042-8. doi: 10.4187/ respcare.06675

\section{FUNDING}

This study was funded by General Projects of Social Development in Shaanxi Province (2019SF-151) and Key Research and Development Project of Shaanxi Province (S2020-YF-ZDCXL-ZDLSF-0048).

\section{ACKNOWLEDGMENTS}

The authors thank all the researchers who created and managed the MIMIC III database.

\section{SUPPLEMENTARY MATERIAL}

The Supplementary Material for this article can be found online at: https://www.frontiersin.org/articles/10.3389/fendo.2020. 611435/full\#supplementary-material

13. Ni YN, Luo J, Yu H, Wang YW, Hu YH, Liu D, et al. Can body mass index predict clinical outcomes for patients with acute lung injury/acute respiratory distress syndrome? A meta-analysis. Crit Care (2017) 21(1):36. doi: 10.1186/s13054-017-1615-3

14. Zhi G, Xin W, Ying W, Guohong X, Shuying L. "Obesity Paradox" in Acute Respiratory Distress Syndrome: Asystematic Review and Meta-Analysis. PloS One (2016) 11(9):e0163677. doi: 10.1371/journal.pone.0163677

15. Soubani AO, Chen W, Jang $\mathrm{H}$. The outcome of acute respiratory distress syndrome in relation to body mass index and diabetes mellitus. Heart Lung (2015) 44(5):441-7. doi: 10.1016/j.hrtlng.2015.06.007

16. Soto GJ, Frank AJ, Christiani DC, Gong MN. Body mass index and acute kidney injury in the acute respiratory distress syndrome. Crit Care Med (2012) 40(9):2601-8. doi: 10.1097/CCM.0b013e3182591ed9

17. Memtsoudis SG, Bombardieri AM, Ma Y, Walz JM, Chiu YL, Mazumdar M. Mortality of patients with respiratory insufficiency and adult respiratory distress syndrome after surgery: the obesity paradox. J Intensive Care Med (2012) 27(5):306-11. doi: 10.1177/0885066611411410

18. Gong MN, Bajwa EK, Thompson BT, Christiani DC. Body mass index is associated with the development of acute respiratory distress syndrome. Thorax (2010) 65(1):44-50. doi: 10.1136/thx.2009.117572

19. Morris AE, Stapleton RD, Rubenfeld GD, Hudson LD, Caldwell E, Steinberg KP. The association between body mass index and clinical outcomes in acute lung injury. Chest (2007) 131(2):342-8. doi: 10.1378/chest.06-1709

20. Brien JMO, Phillips GS, Ali NA, Lucarelli M, Marsh CB, Lemeshow S. Body mass index is independently associated with hospital mortality in mechanically ventilated adults with acute lung injury. Crit Care Med (2006) 34(3):738-44. doi: 10.1097/01.ccm.0000202207.87891.fc

21. Stapleton RD, Suratt BT. Obesity And Nutrition In ARDS. Clin Chest Med (2014) 35(4):655-71. doi: 10.1016/j.ccm.2014.08.005

22. Banack HR, Kaufman JS. The "Obesity Paradox" Explained. Epidemiology (2013) 24(3):461-2. doi: 10.1097/EDE.0b013e31828c776c

23. Banack HR, Stokes A. The 'obesity paradox' may not be a paradox at all. Int J Obes (2017) 41(8):1162. doi: 10.1038/ijo.2017.99

24. De Jong A, Wrigge H, Hedenstierna G, Gattinoni L, Chiumello D, Frat J, et al. How to ventilate obese patients in the ICU. Intens Care Med (2020) 46 (12):2423-35. doi: 10.1007/s00134-020-06286-x

25. Maia LDA, Cruz FF, de Oliveira MV, Samary CS, Fernandes MVDS, Trivelin SDAA, et al. Effects of Obesity on Pulmonary Inflammation and Remodeling in Experimental Moderate Acute Lung Injury. Front Immunol (2019) 10:1215:1215. doi: 10.3389/fimmu.2019.01215

26. Kordonowy LL, Burg E, Lenox CC, Gauthier LM, Petty JM, Antkowiak M, et al. Obesity is associated with neutrophil dysfunction and attenuation of murine acute lung injury. Am J Resp Cell Mol (2012) 47(1):120-7. doi: 10.1165/rcmb.2011-0334OC 
27. Stapleton RD, Dixon AE, Parsons PE, Ware LB, Suratt BT. The association between BMI and plasma cytokine levels in patients with acute lung injury. Chest (2010) 138(3):568-77. doi: 10.1378/chest.10-0014

28. Zhao Y, Li Z, Yang T, Wang M, Xi X. Is body mass index associated with outcomes of mechanically ventilated adult patients in intensive critical units? A systematic review and meta-analysis. PloS One (2018) 13(6):e0198669. doi: 10.1371/journal.pone.0198669

29. Anzueto A, Frutos-Vivar F, Esteban A, Bensalami N, Marks D, Raymondos K, et al. Influence of body mass index on outcome of the mechanically ventilated patients. Thorax (2011) 66(1):66-73. doi: 10.1136/thx.2010.145086

30. Yu Q, Wang D, Wen X, Tang X, Qi D, He J, et al. Adipose-derived exosomes protect the pulmonary endothelial barrier in ventilator-induced lung injury by inhibiting the TRPV4/Ca(2+) signaling pathway. Am J Physiol Lung Cell Mol Physiol (2020) 318(4):L723-41. doi: 10.1152/ajplung.00255.2019

31. Ball L, Serpa Neto A, Pelosi P. Obesity and survival in critically ill patients with acute respiratory distress syndrome: a paradox within the paradox. Crit Care (London England) (2017) 21(1):114-3. doi: 10.1186/s13054-017-1682-5

32. Bime C, Fiero M, Lu Z, Oren E, Berry CE, Parthasarathy S, et al. High Positive End-Expiratory Pressure Is Associated with Improved Survival in Obese
Patients with Acute Respiratory Distress Syndrome. Am J Med (2017) 130 (2):207-13. doi: 10.1016/j.amjmed.2016.09.029

33. De Jong A, Cossic J, Verzilli D, Monet C, Carr J, Conseil M, et al. Impact of the driving pressure on mortality in obese and non-obese ARDS patients: a retrospective study of 362 cases. Intens Care Med (2018) 44(7):1106-14. doi: 10.1007/s00134-018-5241-6

Conflict of Interest: YW was employed by the company Ruibiao (Wuhan) Biotechnology Co.

The remaining authors declare that the research was conducted in the absence of any commercial or financial relationships that could be construed as a potential conflict of interest.

Copyright (c) 2021 Zhang, Wang, Li and Wang. This is an open-access article distributed under the terms of the Creative Commons Attribution License (CC BY).

The use, distribution or reproduction in other forums is permitted, provided the original author(s) and the copyright owner(s) are credited and that the original publication in this journal is cited, in accordance with accepted academic practice. No use, distribution or reproduction is permitted which does not comply with these terms. 